\title{
Linx
}

Revue des linguistes de l'université Paris X Nanterre

38 | 1998

L'acquisition de la temporalité en situation bilingue

\section{Notion de temps et subordination temporelle dans l'acquisition bilingue (français - allemand)}

Temporal notions and temporal subordination in French-German bilingual acquisition

\section{Vera Führer-Nicod}

\section{OpenEdition}

\section{Journals}

Édition électronique

URL : http://journals.openedition.org/linx/867

DOI : $10.4000 / \operatorname{lin} x .867$

ISSN : 2118-9692

Éditeur

Presses universitaires de Paris Nanterre

Édition imprimée

Date de publication : 1 décembre 1998

Pagination : 65-79

ISSN : 0246-8743

\section{Référence électronique}

Vera Führer-Nicod, « Notion de temps et subordination temporelle dans l'acquisition bilingue (français - allemand) », Linx [En ligne], 38 | 1998, mis en ligne le 26 juin 2012, consulté le 19 avril 2019. URL: http://journals.openedition.org/linx/867 ; DOI : 10.4000/linx.867

Ce document a été généré automatiquement le 19 avril 2019

Département de Sciences du langage, Université Paris Ouest 


\section{Notion de temps et subordination temporelle dans l'acquisition bilingue (français - allemand)}

Temporal notions and temporal subordination in French-German bilingual acquisition

Vera Führer-Nicod

\section{Présentation des différentes formes de subordination temporelle de l'allemand et du français}

1 Les conjonctions temporelles les plus fréquemment utilisées en français sont « quand » et «lorsque » qui couvrent à peu près les mêmes champs fonctionnels. Mais le second est beaucoup moins fréquent chez les enfants du fait qu'il s'agit pour l'acquisition principalement d'une langue orale et que «l'input» ne contient en général que la seconde de ces formes.

2 En allemand, ils correspondent à deux lexèmes de fonction bien distincte, distinction liée au temps verbal.

\begin{tabular}{|l|l|}
\hline 1 & « wenn » + présent - quand/lorsque + présent \\
\hline$a$ & (Immer) wenn ich nach Berlin komme, gehe ich in den Zoo. \\
\hline & (Toutes les fois que) quand/lorsque je viens à Berlin, je vais au zoo. \\
\hline & « wenn » + présent - quand/lorsque + futur \\
\hline$b$ & Wenn ich mit meiner Arbeit fertig bin, kann ich ein Buch lesen. \\
\hline
\end{tabular}


Quand /lorsque j'aurai terminé mon travail, je pourrai lire un livre.

3 Dans le deuxième exemple, l'allemand exprime la postériorité rapprochée dans le temps par le présent, tandis que le français utilise le futur.

4 Au passé, il faut distinguer :

\begin{tabular}{|l|l|}
\hline 2 & le fait répété \\
\hline$a$ & Wenn es regnete, blieben wir zu Hause. \\
\hline & Quand/lorsqu'il pleuvait, nous restions à la maison. \\
\hline & le fait unique \\
\hline$b$ & Als sie ankam, war es schon dunkel. \\
\hline & Quand/lorsqu'elle arriva, il faisait déjà nuit. \\
\hline
\end{tabular}

5 Dans les régions du sud de l'Allemagne, le lexème subordonnant « wie » + passé composé prend souvent la place de « als » dans la langue orale.

War es gestern zu spät, wie du in die Schule gekommen bist?

As-tu été en retard hier, quand tu es arrivé à l'école?

6 Il faut mentionner que "wenn » remplit également la fonction conditionnelle, fonction fréquemment utilisée dans les échanges adulte-enfant et qui confère à ce lexème une certaine saillance perceptuelle par rapport à «als ».

$$
\begin{array}{|l|l|}
\hline 4 & \text { Wenn du sofort kommst, fahren wir noch heute nach Berlin. } \\
\hline & \text { Si tu viens tout de suite, nous partirons aujourd'hui même pour Berlin. } \\
\hline
\end{array}
$$

7 En allemand et en français, il existe bien sûr d'autres conjonctions de subordination temporelle, comme p. ex.

\begin{tabular}{|l|l|}
\hline während & pendant que \\
\hline \hline bevor & avant que/avant de \\
\hline nachdem & après que/après avoir \\
\hline
\end{tabular}


que nous ne traiterons que peu ici, car les enfants que nous avons observés préfèrent dans ces cas deux propositions principales coordonnées reliées par «und» et/ou des adverbes temporels, plutôt qu'une structure hypotaxique.

\begin{tabular}{|l|l|}
\hline 5 & Ich esse und du liest mir etwas vor. \\
\hline & Je mange et tu me lis quelque chose. \\
\hline & Ich esse zuerst und danach liest du mir etwas vor. \\
\hline & Je mange d'abord et après/ensuite tu me lis quelque chose. \\
\hline
\end{tabular}

au lieu de

Après avoir fini de manger, tu me liras une histoire.

8 Il est d'ailleurs fréquent que « l'input » ne contienne pas non plus ce type de structures étant donné le caractère oral et familier du contexte.

En allemand, la subordination est soumise à une contrainte syntaxique qui n'existe pas en français et qui nous intéressera par la suite dans l'acquisition bilingue. Dans les cas où la subordonnée vient en tête de l'énoncé, sujet et verbe de la proposition principale sont inversés, les deux verbes conjugués sont donc contigus, tandis qu'en français l'ordre des mots est maintenu.

\begin{tabular}{|l|l|}
\hline 6 & Als der Zug anhielt, war es schon zu spät. \\
\hline & Lorsque le train s'est arrêté, il était (V Pro) déjà trop tard. \\
\hline
\end{tabular}

10 Une seconde différence syntaxique réside dans le fait que lorsqu'on ajoute d'autres éléments aux subordonnées, l'unité sujet-verbe conjugué est brisée en allemand, la forme fléchie du verbe occupant la place finale de l'énoncé.

$$
\begin{array}{|l|l|}
\hline 7 & \text { Als der Zug auf Bahnsteig } 5 \text { anhielt, war es schon zu spät. } \\
\hline \hline & \text { Lorsque le train s'arrêta au quai 5, il était déjà trop tard. } \\
\hline
\end{array}
$$

11 Pour l'enfant bilingue il s'agit par conséquent, sur le plan linguistique, non seulement d'acquérir les connecteurs subordonnants, mais également les temps verbaux et les structures syntaxiques appropriés à chacune de ses langues.

\section{Les phases de l'acquisition linguistique en général}

12 Les trois stades du développement linguistique parcourus par l'enfant sont bien connus, et nous ne ferons que les rappeler brièvement. L'enfant s'exprime d'abord en 
holophrases, pour passer vers l'âge de $1 ; 6$ à 2 ans aux énoncés à deux termes. Le stade qui nous intéresse particulièrement pour l'acquisition de la subordination est le troisième qui débute selon les enfants vers $2 ; 6$ à 3 ans. L'enfant construit alors des énoncés à trois éléments et plus, et commence à établir des relations grammaticales entre les éléments. A l'intérieur de chaque stade, l'apprenant unilingue ou bilingue traverse d'autres phases pour différents objets linguistiques, décrites par des chercheurs comme C. et W. Stern (1928), T. Taeschner (1983), J. Petit (1987) et N. Müller (1995).

Tout d'abord, l'enfant, pendant quelque temps, reproduit des formes correctes entendues dans son entourage et mémorisées.

(la phase d'organisation où l'enfant établit une hypothèse concernant l'emploi d'un morphème ou d'un lexème qu'il met à l'essai en généralisant au maximum. Il l'étend au-delà des limites reconnues par la langue standard. Cette attitude acquisitionnelle est universelle et présente une spontanéité que le terme d'hypothèse ne traduit pas tout à fait. Cette période peut s'étendre sur plusieurs semaines à plusieurs mois.

A la troisième phase, l'hypothèse erronée s'approche petit à petit du standard, mouvement inverse de restriction de généralisation, souvent déclenché par l'usage correct de l'entourage ("modelling», selon les expériences menées par C. Cazden en 1968), associé à une prise de conscience linguistique progressive de l'enfant.

Ces phases successives se retrouvent pour la plupart des phénomènes linguistiques, et nous les illustrons ci-dessous pour ce qui est de l'acquisition des subordonnées temporelles.

\section{L'évolution chronologique des subordonnées chez l'enfant (avec un regard privilégié sur les subordonnées temporelles)}

17 La notion de temps, la situation de l'individu dans le temps, la relation de cause à effet, ne sont pas des données immédiates de la conscience de l'enfant. Leur conceptualisation nécessite une longue expérimentation sur le monde qui ne s'achève qu'entre 7 et 9 ans.

18 A l'âge de 5 ou 6 ans, l'enfant ne maîtrise pas encore les mesures conventionnelles du «temps social», p. ex. l'heure, et il n'est pas rare qu'il interroge l'adulte pour se situer dans le temps afin d'acquérir des points d'ancrage précis. Par exemple à onze heures du matin, un de nos sujets observés (6 ans) demande si « c'est l'après-midi ou déjà le soir ». Il ne s'agit pas d'un problème lexical, l'enfant ayant montré par ailleurs qu'il disposait activement des lexèmes appropriés. Mais il n'a pas encore acquis toute la gamme des références temporelles.

19 Aussi l'expression linguistique des relations entre l'individu et le monde ou entre plusieurs événements, comme l'acquisition des propositions subordonnées et de leurs conjonctions, dépend-elle non seulement de l'évolution linguistique de l'enfant, mais également de son développement cognitif. Complexité cognitive et complexité linguistique de chaque langue se conditionnent mutuellement.

20 Certains travaux, la plupart portant sur des enfants unilingues (allemand ou français), décrivent le déroulement chronologique dans l'acquisition des subordonnées. Pour l'allemand, C. et W. Stern observent en 1928 que leur fils Günther construit des 
subordonnées complétives nettement plus tard que les autres subordonnées. Pour le français, Clark (1985) relève les mêmes résultats. En ce qui concerne l'acquisition bilingue, Kielhöfer \& Jonekeit (1983), N. Müller (1993) et nous-même (1992/1994) constatons que l'enfant bilingue aussi bien que l'enfant unilingue allemand ou français n'emploient « daß » et/ou " que » qu'après beaucoup d'autres conjonctions, et ce au plus tôt vers l'âge de $3 ; 6-4 ; 0$. Par contre, la fille aînée des Stern utilise des constructions hypotaxiques reliées par «daß» dès l'âge de $3 ; 2$, mais également après d'autres subordonnants. Ces observations nous rappellent qu'il est difficile de fixer l'âge de l'acquisition des subordonnées, et que beaucoup de facteurs individuels et extralinguistiques entrent en jeu dans le rythme de l'acquisition (situation de l'enfant dans la famille, temps consacré directement à l'enfant etc.).

Il nous semble par conséquent plus utile de présenter l'acquisition de la subordination dans son déroulement chronologique plutôt que de fixer des tranches d'âge qui peuvent différer grandement d'un enfant à l'autre.

Kielhöfer \& Jonekeit (1985: 52) définissent une suite acquisitionnelle pour certaines conjonctions subordonnantes en fonction de l'évolution cognitive de l'enfant.

Tout d'abord, l'enfant doit comprendre que les événements peuvent se suivre dans le temps (temporalité). Ensuite, il saisit qu'une action antérieure peut conditionner une action postérieure (causalité). Projetée dans le futur, cette compréhension ouvre la voie de la finalité. Concession et irréalité (condition) sont également étroitement liées à la causalité, et leur acquisition dépend de sa compréhension antérieure.

Lorsqu'à $2 ; 3$ on peut entendre "si tu renverses, moi je vais donner fessée », Kielhöfer indique que la conjonction prend ici une fonction pragmatique (mise en garde, menace) puisque son contenu sémantique n'est pas encore acquis par l'enfant.

Toutes les études sur des enfants unilingues, pour d'autres langues aussi (p. ex. pour l'anglais Bloom et al. 1980 cités par Müller 1993 : 87), concordent en ce qui concerne les deux premiers types de subordonnées.

Il est intéressant de constater que les conjonctions adverbiales avec fonction sémantique (quand, parce que) apparaissent avant les conjonctions occupant purement une fonction syntaxique (que). Même si l'expression de la fonction temporelle semble souvent le premier type de subordonnée selon la plupart des travaux, N. Müller $(1993: 87)$ a observé chez son sujet bilingue une utilisation plus précoce de "parce que » en français, tandis qu'en allemand «wenn » (quand) est apparu avant « weil » (parce que).

Nos propres observations sur des sujets bilingues ont montré que les subordonnées causales en français d'abord, en allemand ensuite, apparaissent quelques semaines avant la temporalité exprimée par "wenn », qui est tardivement complétée par l'apparition de "als» (quand+passé). Dans tous les cas, l'expression de la temporalité et celle de la causalité sont étroitement liées dans le développement cognitif et l'acquisition linguistique de l'enfant. L'indication d'un ordre de succession par rapport à un temps différent de celui de l'énonciation peut s'organiser autour d'une relation causale chez l'un, par une relation temporelle chez l'autre sujet. Nous verrons plus loin en détail le processus acquisitionnel.

28 L'apparition de subordonnées conditionnelles constitue la troisième étape. Le système syntaxique de l'allemand semble constituer une aide à l'acquisition, car la conjonction "wenn", maniée depuis longtemps par l'enfant, couvre également cette fonction. L'acquisition des temps et modes verbaux appropriés prendra plus de temps. 
La quatrième fonction utilisée par l'enfant bilingue est la fonction complétive, que Kielhöfer \& Jonekeit ne mentionnent pas dans leur tableau.

La subordonnée finale apparaît en dernier vers l'âge de 7 ans où nos observations se terminent. A cet âge, la conjonction allemande « damit » (français langue forte) n'est pas encore utilisée par tous les enfants, mais exprimée par un substitut, tandis que «pour que » + subjonctif existe dans la langue forte (cf. Führer-Nicod $1994: 228$ sqq. pour plus de détails).

\begin{tabular}{|l|l|}
\hline 9 & Je te donne ces images pour que tu les colles. (Vic $6 ; 8)$ \\
\hline & pour que la directrice sache. (Vic $6 ; 8)$ \\
\hline & Du tust Alkohol (hin), zum das weggeht? (Vic $6 ; 9)$ \\
\hline & Tu mets de l'alcool pour que cela parte (une tache)? \\
\hline
\end{tabular}

31 Dans cette section, nous avons vu que l'acquisition des différentes fonctions de subordination est en relation étroite avec l'évolution cognitive de l'enfant. L'expression linguistique de la subordination, aussi déviante de la langue standard qu'elle soit au début, s'appuie sur un développement cognitif préalable.

En ce qui concerne le déroulement chronologique de l'acquisition de la subordination, la plupart des recherches menées dans ce domaine montrent que l'acquisition bilingue suit dans l'ensemble les étapes décrites chez l'enfant unilingue, et elles placent les subordonnées temporelles et causales au début de l'acquisition.

Après une brève présentation du corpus, nous nous interrogerons dans la section suivante plus particulièrement sur leur développement dans l'acquisition précoce des deux langues allemande et française, ainsi que sur les phénomènes de dominance qui pourraient se répercuter sur cette acquisition.

\section{L'acquisition des subordonnées temporelles (et causales) par l'enfant bilingue français-allemand}

Nos observations dans le domaine des subordonnées portent principalement sur les productions de deux enfants bilingues entre l'âge de 1 an et 7 ans pour l'un (Vic) et entre $3 ; 5$ et $4 ; 5$ pour l'autre (Ch). Les deux enfants vivent en France dans des familles bilingues, et leur langue forte, notamment depuis l'entrée à l'école, est celle de l'environnement, le français. Les travaux de C. et W. Stern pour l'allemand, de F. François pour le français, ainsi que de nombreuses études sur des sujets bilingues nous serviront de comparaison.

Dans l'élaboration de son système linguistique de subordination, l'enfant bilingue français - allemand passe par plusieurs phases. Tantôt les deux langues évoluent simultanément, tantôt l'une précède, voire influence l'autre.

Nos observations dans ce qui suit adoptent un point de vue purement linguistique. 


\section{Première phase : sans connecteur, structure parataxique}

Avant d'établir une relation syntaxique explicite entre les éléments, l'enfant exprime le rapport entre deux événements sous la forme parataxique dans un énoncé à deux parties non séparées par une pause.

\begin{tabular}{|l|l|}
\hline 10 & is nich da schieben, kann nich mahle mahle \\
\hline & Weil der « Breisschieber » nicht da ist, kann ich nicht umrühren. \\
\hline & Parce que « la cuillère» n'est pas là, je ne peux pas tourner (ma bouillie). \\
\hline
\end{tabular}

Chez les enfants bilingues observés par N. Müller en Allemagne et par nous-même en France, les subordonnées les plus précoces sont des énoncés causaux en français, rapidement suivis par ceux en allemand ainsi que par des énoncés temporels. Tous se distinguent de la langue adulte par l'absence du connecteur ainsi que, parfois, par l'absence du verbe conjugué.

\begin{tabular}{|l|l|}
\hline 11 a & / Ivar I-Ivar groß / Ivar die Hose / Ivar groß (wenn, ist) ( 2 ;11) \\
\hline & / Ivar grand, I. le pantalon, / I. grand (quand, sera) \\
\hline b & ça c'est pour le soleil / il fait du soleil (quand) $(3 ; 1)$ \\
\hline c & und / da sind (n)öwe eh eh de ca(s) sind zu (wenn, Löwen, cage) (3;4) (Müller, ibid.. 197) \\
\hline \hline & et / des lions seront là, (quand) les cages seront fermées \\
\hline
\end{tabular}

Nous supposons que le choix de la langue dans laquelle débute l'acquisition des subordonnées n'est pas aléatoire. La complexité moindre du français par rapport à la difficulté syntaxique de l'ordre des mots en allemand semble jouer un rôle. Cette hypothèse se trouve confirmée dans les exemples de Kielhöfer \& Jonekeit, car l'un des deux enfants observés en Allemagne forme déjà des énoncés causaux corrects en français, tandis qu'en allemand il en est encore au stade des précurseurs de la subordination.

\begin{tabular}{|l|l|}
\hline 12 & donne à boire pas (pasque) que j'ai soif! $(3 ; 0)$ \\
\hline & Gib zu trinken / ich hab Durst. $(3 ; 0)$ Kielhöfer \& Jonekeit : 51) \\
\hline
\end{tabular}

40 Taeschner (1983) mentionne brièvement que l'apparition de structures subordonnées chez les deux enfants bilingues italien-allemand a été observée au même moment que chez les enfants unilingues, mais que les énoncés italiens étaient corrects beaucoup plus tôt. Dans son corpus, nous avons trouvé les exemples suivants : 


\begin{tabular}{|l|l|}
\hline $13 a$ & Jetzt ist dunkel, spielen da $(3 ; 0)$ \\
\hline \hline & Weil es jetzt dunkel ist, spiele ich da. \\
\hline & Maintenant (parce qu'il fait) nuit, jouer là \\
\hline \hline$b$ & Perché io voglio fare una barchetta $(2 ; 8)$ \\
\hline & Parce que je veux faire un bateau \\
\hline
\end{tabular}

41 Nous ne trouvons que peu d'exemples pour cette période dans les études bilingues. La raison en est sans doute qu'elle est de courte durée, et que les énoncés sans connecteur coexistent rapidement avec les premiers essais de subordonnants.

\section{Deuxième phase : apparition de la structure hypotaxique, apparition des connecteurs}

42 On peut distinguer dans cette phase plusieurs étapes successives, qui ont été relevées dans différents travaux. Mais la durée des étapes varie selon les sujets. Dans tous les cas, cette deuxième grande phase se termine pour les sujets bilingues avec l'allemand langue faible par la production correcte de subordonnées temporelles et causales en français. En allemand, l'ordre des mots et le choix de certains connecteurs dévient encore de la langue adulte. Pour le sujet unilingue, cette phase se concrétise la plupart du temps par l'acquisition pleine des structures en question.

43 Stern et Stern (p. 247) remarquent chez leur fils que le passage de la structure parataxique à la structure hypotaxique constitue un pas supplémentaire vers la subordination complexe :

$$
\begin{array}{|l|l|}
\hline 14 & \text { hilde groß is, großes stück essen. ich klein bin }(2 ; 7) . \\
\hline & \text { Hilde est grande, manger grand morceau. je suis petit } \\
\hline
\end{array}
$$

44 Malgré l'absence de la conjonction «weil/parce que» et du verbe fléchi dans la proposition principale, l'ordre des mots montre clairement la prise de conscience linguistique progressive de l'enfant. Chez lui, la place finale occupée par le verbe fléchi dans la proposition subordonnée (is) constitue un marqueur fonctionnel de la subordination.

Pour les sujets bilingues, ce problème est plus complexe (nous y reviendrons) et nous n'avons trouvé qu'un seul exemple dans N. Müller (p. 196) :

$$
\begin{array}{|l|l|}
\hline 15 & \text { und / sie gar nicht da sind (wenn) }(3 ; 1) \\
\hline & \text { et / ils ne seront pas là (quand) } \\
\hline
\end{array}
$$


voivante voit apparaître une ébauche de connecteur, qui se différencie d'ailleurs souvent selon les différentes fonctions à accomplir. Dans les études sur l'acquisition du langage, bilingue ou non, les phénomènes d'ébauche n'ont pas toujours été mis en évidence. Ils nous semblent néanmoins importants à prendre en compte car ils sont l'expression de la reconnaissance par l'enfant de la catégorie fonctionnelle assumée par les connecteurs.

Enfant unilingue :

48 Trois mois plus tard une autre ébauche de connecteur, [mm], remplit la même fonction de substitut pour l'expression de la finalité. C'est seulement vers $4 ; 7$ que les parents de Günther Stern notent un énoncé causal complet.

Enfant bilingue :

17 je va je va demander maman (e) (e) (=parce que) je sais plus (Ivar 2 ;1) (Müller, p. 197)

La dernière étape de cette deuxième grande phase voit s'établir petit à petit par un double mouvement d'extension et de réduction l'utilisation correcte des subordonnants.

Kielhöfer \& Jonekeit (p. 51), Müller (p. 195) et nous-même (1994) avons observé que la première apparition d'un connecteur s'effectue dans les énoncés français, que cette langue soit la langue forte ou la langue faible de l'enfant. Peu de temps après, l'autre langue suit cette évolution.

\begin{tabular}{|l|l|}
\hline $18 a$ & donne à boire pasque j'ai soif! (Jens $3 ; 0)$ \\
\hline \hline$b$ & parce que y a des poules / (qui) ils mordent ils mordent les chevaux $(2 ; 9)$ \\
\hline$c$ & je mange pas ça parce que c'est trop chaud (Vic $2 ; 9)$ \\
\hline
\end{tabular}

51 Pour l'évolution subséquente, Kielhöfer \& Jonekeit ne donnent pas d'exemple concret, mais ils indiquent qu'à $3 ; 2$ Jens forme des énoncés causaux et temporels corrects dans ses deux langues.

19a wei(n) eh wi(n) eh weil meine mama war nich da $(3 ; 0)$ 


\begin{tabular}{|l|l|}
\hline & par pa parce que ma maman était pas là \\
\hline \hline$b$ & die Sonne is weg die kann nicht mehr kommen weil es ist Schnee (Vic $3 ; 5)$ \\
\hline & le soleil est parti il ne peut plus revenir parce qu'il y a de la neige. \\
\hline
\end{tabular}

Peu de temps après, avec quelques différences individuelles, apparaissent les premières structures temporelles. D'après nos propres observations cela se passe d'abord dans la langue forte de l'enfant, ou bien simultanément dans ses deux langues. La notion connecteur - fonction subordonnante a été acquise par l'enfant d'abord pour les énoncés causaux, et est aussitôt transposée à l'expression de la temporalité.

\begin{tabular}{|l|l|}
\hline 20a & erst wenn wir sind fertig mit das (Jvar $3 ; 4)$ \\
\hline & seulement quand nous aurons fini avec ça \\
\hline b & jetzt wenn wir sind aufgestanden $(3 ; 11)$ \\
\hline & maintenant quand nous nous sommes levés \\
\hline c & daß die fische sind tot wenn die sind ra-raus ne ? ( 433$)($ Müller, 206) \\
\hline & que les poissons sont morts quand ils sont dehors hein? \\
\hline
\end{tabular}

Le corpus de Müller ne nous donne pas d'exemple pour la langue faible.

\begin{tabular}{|l|l|}
\hline $21 a$ & après quand je serai habillée, est-ce que je pourrai regarder la télé ? (Vic $3 ; 6)$ \\
\hline \hline$b$ & Quand il sera pas là ... (Vic $4 ; 0)$ \\
\hline$c$ & Vous faites ça quand vous êtes guéris. (Vic $4 ; 2)$ \\
\hline
\end{tabular}

54 Le dernier exemple est étonnant, car l'enfant a prouvé de nombreuses fois auparavant qu'il maîtrisait l'exigence du français d'utiliser une forme verbale du futur pour exprimer un événement postérieur au moment de la parole. Il est nécessaire de considérer le contexte situationnel (l'enfant observe ses parents qui sont en train de se gargariser pour soigner leur mal de gorge) pour comprendre que "quand " prend ici le sens de "jusqu'à ce que ». Une utilisation presque correcte de ce dernier connecteur n' a été remarquée qu'à l'âge de $6 ; 11$ sous la forme de « jusqu'à que ».

Ce phénomène d'extension d'un seul signifiant à plusieurs fonctions n'est pas rare dans l'acquisition linguistique en général, mais se produit chez notre sujet en plus grand nombre dans la langue faible. Dans le cas de la temporalité, il s'agit pendant longtemps du même lexème "wenn" (quand), qui occupe également la fonction de "als", c.à.d. la relation temporelle entre des faits singuliers du passé. Il faut certainement voir une des raisons de cette extension dans la grande fréquence de ce connecteur en allemand. D'autres explications pourraient être soit le fait que l'enfant possède certes la maturité 
cognitive pour se référer à un événement antérieur au moment de la parole (il forme correctement le temps verbal), mais qu'il n'a pas encore réalisé que l'allemand exige un connecteur spécifique dans ce cas, soit un manque d'input.

Il faut par conséquent être prudent et ne pas conclure immédiatement à un phénomène d'influence de la langue forte où le même signifiant « quand» remplit les deux fonctions. D'autres déviances concernant les subordonnants sont relevées également chez d'autres enfants bilingues pour un contexte identique (Ronjat (1913), Führer-Nicod, 1994), mais l'extension concerne le subordonnant "wie» (comme, comment, que) qui ne peut s'expliquer en relation avec la deuxième langue des sujets.

57 Voici l'illustration de nos propos, d'abord par l'utilisation correcte du connecteur «wenn » (22) et ensuite par son extension (23):

\begin{tabular}{|c|c|}
\hline $22 a$ & Nachher wenn ich habe geholt meine Bonbons. (Vic $3 ; 9)$ \\
\hline & Après/tout à l'heure quand j'aurai été chercher mes bonbons \\
\hline \multirow[t]{2}{*}{$b$} & Aber wenn wenn ich bin einmal gro $\beta($ Vic $4 ; 0)$ \\
\hline & Mais quand quand je serai grande \\
\hline \multirow[t]{2}{*}{$c$} & Aber wenn ich geh in die Schule, ich seh den Mond. (Vic $4 ; 2)$ \\
\hline & Mais quand je vais à l'école, je vois la lune. \\
\hline \multirow[t]{4}{*}{$d$} & $\begin{array}{l}\text { wenn man blau / (hat) und dann ham wir die Schnecke und dann nimmt man eine Karte (Charl. } \\
3 ; 11 \text { ) }\end{array}$ \\
\hline & quand on / (a) bleu et puis nous avons l'escargot et puis on prend une carte \\
\hline & wenn der Bubi ist lieb (Ronjat, fréquent avant $3 ; 3$ ) \\
\hline & quand Bubi est gentil \\
\hline \multirow[t]{2}{*}{$f$} & wenn du vormittags hast einen fürchterlichen Hunger, dann nimmst du dir eins. (Ronjat $4 ; 0)$ \\
\hline & quand le matin tu as une terrible faim, tu en prends un. \\
\hline \multirow[t]{2}{*}{$23 a$} & Wenn ich war klein schon $(=$ als $)($ Vic $4 ; 1)$ \\
\hline & quand j'étais déjà petite \\
\hline$b$ & Wenn ich war ein Babies, dann ich hab mich immer untern Tisch gesessen. $(=a l s)($ Vic $4 ; 1)$ \\
\hline & Quand j'étais un bébé, alors je me suis toujours *assite sous la table. \\
\hline
\end{tabular}

Deux jours après un input direct de la part du locuteur adulte, l'enfant essaie la nouvelle forme als, mais se trompe de signifié : 


\begin{tabular}{|l|l|}
\hline$c$ & Als eine Serviette nehmen, wollt ich eine Hose. $(=$ anstatt $)($ Vic $5 ; 9)$ \\
\hline & Quand prendre une serviette, je voulais un pantalon. ( = au lieu de) \\
\hline$d$ & das ist dich, wenn du warst ein kleiner Junge ( = als) (Leopold $5 ; 3)$ \\
\hline & ça c'est toi, quand tu étais un petit garçon \\
\hline e & Und wenn ich das gedrückt hab, weil es klein war, ist es groß / (geworden). (Vic $6 ; 8)$ \\
\hline & Et quand j'ai appuyé sur ça, parce que c'était petit, c'est (devenu) grand. \\
\hline
\end{tabular}

Ensuite l'exploration se poursuit, le signifiant change selon l'enfant, mais aussi chez le même sujet :

\begin{tabular}{|c|c|}
\hline $24 a$ & Vorhin, daß ich das gesagt hatte $(=a l s)($ Vic $6 ; 9)$ \\
\hline & Tout à l'heure, que j'avais dit cela (=quand) \\
\hline \multirow[t]{2}{*}{$b$} & Wie ich, wie ich Baby war, da hab ich immer so gemacht $(=a l s)($ Charl. $3 ; 11)$ \\
\hline & comme je, comme j'étais bébé, alors j'ai toujours fait comme ça (=quand) \\
\hline \multirow[t]{2}{*}{$c$} & Wie der Papa war so klein $(=$ als) (Ronjat $3 ; 3)$ \\
\hline & Comme papa était aussi petit \\
\hline \multirow[t]{2}{*}{$d$} & wenn ich klein war, ich hab getanzt $(=$ als $)($ Giulia $3 ; 2)$ \\
\hline & quand j'étais petite, j'ai dansé \\
\hline \multirow[t]{2}{*}{$e$} & wo ich klein war ich bin in Brasilien gegangen $(=$ als $)($ Giulia $3 ; 2)($ Taeschner $p .164)$ \\
\hline & où j'étais petite, suis je allée dans Brésil \\
\hline
\end{tabular}

60 A l'âge de sept ans, où notre corpus s'arrête, l'enfant Vic est toujours à la phase de surextension, à la recherche du signifiant approprié pour verbaliser un événement unique antérieur. Le mouvement réducteur a déjà eu lieu en ce qui concerne le connecteur "wenn" et continuera pour les autres jusqu'au moment où signifié et signifiant concorderont avec leurs correspondants dans la langue adulte.

61 Cette dernière étape révèle une autre grande difficulté, à laquelle sont confrontés tous les sujets bilingues français-allemand : la place du verbe fléchi dans les énoncés subordonnés allemands. Ce problème sera résolu à la phase suivante. 


\section{Troisième phase : acquisition de la place correcte du verbe fléchi}

62 Les exemples de la phase précédente ont montré que l'enfant bilingue ne parvient qu'après de nombreux hésitations et essais à réaliser une structure subordonnée correspondant à la langue adulte en allemand, c'est à dire où le verbe fléchi occupe la dernière place de la proposition subordonnée (Vfinal) et où le sujet et le verbe sont inversés dans la proposition principale.

Ce ne sont d'ailleurs pas seulement des sujets bilingues français-allemand qui se trouvent confrontés à cette difficulté. Taeschner (italien-allemand) et Leopold (anglais-allemand) ont observé des problèmes analogues. La raison principale de cette difficulté dans l'ordre des mots n'est pas non plus due au fait que pour tous ces enfants l'allemand soit la langue faible. Ronjat (ex. 22) ainsi que le corpus de Müller (ex. 20) enregistré dans un environnement germanophone font état des mêmes obstacles.

En ce qui concerne l'acquisition unilingue de la subordination en allemand, plusieurs études tendent à démontrer que Vfinal est acquis immédiatement (Clahsen, Stern \& Stern 1981). D'autres pourtant donnent des exemples contraires (cf. Müller pp. 87-95 pour plus de détails). Il semblerait donc qu'un processus hésitant et plein de déviances soit le cas général pour l'acquisition bilingue, tandis que pour l'acquisition unilingue des difficultés de cet ordre constituent plutôt une exception.

Quelles sont alors les raisons de cette difficulté acquisitionnelle?

Nous avons constaté chez l'enfant Vic qu'un séjour en Allemagne avait considérablement accéléré la mise en place de l'ordre allemand des mots. Une immersion régulière dans la langue faible est donc nécessaire pour réduire une éventuelle extension de structures françaises. D'un autre côté, les expériences linguistiques du sujet se sont multipliées : entre l'âge de 4 ans $/ 4 ; 6$ et 5 ans, l'inversion dans l'interrogation et après certains constituants placés en tête dans l'énoncé allemand se met en place (Führer-Nicod 1994 : 461-4).

67 L'inversion dans la proposition principale précède souvent Vfinal qui représente une difficulté supplémentaire (voir les ex. $22 \mathrm{~d}$ et $\mathrm{f}$ ), car sujet et verbe fléchi doivent se séparer. Taeschner (p. 165) explique cette difficulté à placer le verbe fléchi en position finale de la subordonnée en proposant que sujet et verbe forment un ensemble inséparable, une entité globale acquise par l'enfant durant la première période de son acquisition.

68 N. Müller (p. 256) a constaté chez l'un de ses sujets que la mise en place de Vfinal ne s'effectuait pas globalement, mais séparément pour chaque connecteur.

Dans tous les cas, cette nouvelle structure n'apparaît pas tout d'un coup chez l'enfant, mais coexiste pendant plusieurs mois en nombre croissant avec des formes erronées. Certains sujets traversent même une brève période préliminaire durant laquelle le verbe fléchi est supprimé traduisant bien, à notre avis, la prise de conscience linguistique progressive de l'apprenant bilingue et quelquefois unilingue.

\begin{tabular}{|l|l|}
\hline $25 a$ & $\begin{array}{l}\text { wenn man blau / (hat) und dann ham wir die Schnecke und dann nimmt man eine Karte (Charl. } \\
3 ; 11) \quad(\text { exemple déjà cité }: 22 \text { d) }\end{array}$ \\
\hline & quand on / (a) bleu et puis nous avons l'escargot et puis on prend une carte \\
\hline
\end{tabular} 
Du wenn wenn du dann gefangen (hast) / faut nicht nicht da gehn (Vic 4 ;0)

Tu quand quand tu (as) alors attrapé faut pas pas aller là observateurs constatent une majorité de productions correspondant à la langue adulte (environ $93 \%$ entre $4 ; 11$ et $5 ; 2$ d'après N. Müller p. 226).

\begin{tabular}{|l|l|}
\hline $27 a$ & wenn - hm wenn mein bruder, öhm die nacht, sich aufwacht u und schreit ? (Ivar $5 ; 0)$ \\
\hline & réveille et crie la nuit? \\
\hline$b$ & wenn wenn mama wieder da is hat er ein bobo dann ne ? (Ivar $5 ; 2)$ \\
\hline & quand quand maman sera de retour, il a alors un bobo, hein ? \\
\hline$c$ & Wenn ich gute Noten hab, krieg ich Glitzerknetgummi. (Vic $6 ; 8)$ \\
\hline \hline & Quand j'aurai de bonnes notes, j'aurai de la pâte à modeler brillante. \\
\hline
\end{tabular}

\section{Conclusions}

73 Le but de notre étude était d'abord de situer l'apparition des subordonnées temporelles dans l'évolution cognitive de l'enfant. Nous avons constaté que la conceptualisation du temps et la réalisation linguistique des relations entre événements sont soumis à un long processus d'évolution qui s'étend de trois à sept ans.

Nous nous sommes intéressée ensuite à l'acquisition linguistique de ces énoncés par des enfants bilingues. Les deux premières des trois grandes phases, à savoir: 1) sans connecteur, structure parataxique ; 2) connecteurs, structure hypotaxique ; 3) ordre des mots (place du verbe fléchi), sont parcourues non seulement par les enfants bilingues, mais également par les enfants unilingues. Certains sujets unilingues s'engagent même dans la troisième phase avant de pouvoir réaliser des énoncés à subordination corrects en allemand.

Nous avons observé pendant la deuxième phase que la dynamique acquisitionnelle se traduit chez certains sujets par l'extension du connecteur « wenn » à la fonction d'autres 
subordonnants temporels (surtout «als »). Ce phénomène n'existe pas seulement dans l'acquisition bilingue français-allemand, mais il est rapporté également pour des sujets bilingues anglais-allemand et italien-allemand. Aussi bien en français avec "quand» qu'en anglais avec "when", un seul connecteur remplit les fonctions de l'allemand "wenn" vs «als ». Sans négliger l'importance des facteurs évoqués pour cette deuxième phase (fréquence des occurrences en allemand, manque d'input), nous suggérons d'y voir l'influence de la langue forte et un transfert négatif.

deuxième domaine éventuellement assujetti aux influences et transferts négatifs de la langue forte sur la langue faible est l'ordre des mots et notamment la place du verbe fléchi. Tous les enfants bilingues rencontrent des difficultés à ce sujet dans les subordonnées allemandes. Mais nos recherches ont montré que ce n'est pas tant la structure sujet + verbe conjugué de la langue forte qui influence cette acquisition, car le phénomène est identique pour les enfants ayant l'allemand pour langue forte ou langue faible. L'enfant doit d'abord prendre conscience que verbe fléchi et sujet ne forment pas un bloc insécable (ce qui peut être considéré comme acquis lorsque le verbe est supprimé dans la subordonnée en allemand, et parfois même dans la langue qui ne connaît pas ce déplacement (cf. ex. 25 et 26), ce qui précède la capacité à déplacer le verbe fléchi à l'intérieur de la construction.

L'acquisition des temps verbaux se comportant différemment dans leurs deux langues (présent / futur) ne semble pas poser de problème aux enfants bilingues françaisallemand.

On peut conclure de ces ensembles de faits que le transfert négatif d'éléments de la langue forte vers la langue faible touche l'acquisition des structures subordonnées dans une mesure bien moindre qu'il n'y paraît au premier abord.

\section{BIBLIOGRAPHIE}

FRANÇOIS Frédéric, FRANÇOIS Denise, SABEAU-JOUANNET Emilie, SOURDOT Marc (1977) : La syntaxe de

l'enfant avant 5 ans. Larousse, Paris.

FÜHRER-NICOD, Vera (1994): Recherches sur le bilinguisme franco-allemand chez les jeunes enfants.

Presses Universitaires de Reims.

FÜHRER-NICOD, Vera (1995) : Beobachtungen zum Erwerb der Interrogativstrukturen beim doppelten Erstsprachenerwerb von Deutsch und Französisch. In : E. Faucher, R. Métrich \& M. Vuillaume (Hrsg.) : Signans und Signatum, Festschrift für Paul Valentin zum 60. Geburtstag. Gunter Narr, Tübingen.

KIELHÖFER Bernd \& JONEKEIT Sylvie (1985) : Zweisprachige Kindererziehung. 3e édition, Stauffenberg, Tübingen.

KLEIN, Wolfgang (1992) : Zweitspracherwerb. 3e édition, Athenäums Studienbuch, Anton Hain, Frankfurt-am-Main. 
LEOPOLD, Werner F. (1949): Speech development of a bilingual child. A linguistic's record, quatre volumes, Illinois.

MEISEL, Jürgen M. (ed.) (1990) : Two first languages : Early Gram-matical Development in Bilingual Children. Foris, Dordrecht.

MÜLLER, Natascha (1993) : Komplexe Sätze. Der Erwerb von COMP und von Wortstellungsmustern bei bilingualen Kindern (Französisch / Deutsch). Gunter Narr, Tübingen.

PETIT, Jean (1987) : Acquisition linguistique et interférences. APLV, Paris.

RONJAT, Jules (1913) : Le développement du langage observé chez un enfant bilingue. Champion, Paris.

STERN, Clara et William (1981) : Die Kindersprache. Réédition de la quatrième édition de 1928, Wissenschaftliche Buchgesellschaft, Darmstadt.

TAESCHNER, Traute (1983) : The sun is feminine. Springer, Berlin.

\section{RÉSUMÉS}

L'article met en parallèle l'évolution cognitive de l'enfant, sa conceptualisation du temps, avec l'apparition des subordonnées et leurs réalisations linguistiques entre trois et sept ans. Les premières subordinations réalisées sont temporelles et causales - de même que les premiers subordonnants.

L'acquisition bilingue de ces types de subordonnées est ensuite détaillée, en trois phases :

1) sans connecteur, structure parataxique ;

2) avec connecteur, structure hypotaxique, phases par lesquelles passent également les enfants unilingues. La phase 2) voit l'utilisation adéquate de subordonnées temporelles et causales en français, langue forte ou faible de l'enfant. En allemand, l'ordre des mots et l'emploi de certains connecteurs s'écartent encore de la langue adulte. Les connecteurs font également l'objet $\mathrm{d}^{\text {c }}$ "ébauches" et de sur-extensions.

3) le placement adéquat du verbe fléchi en allemand est finalement acquis.

On en conclut que l'influence de la langue forte de l'enfant bilingue sur sa langue faible est moins importante qu'on ne tend à le penser.

The article relates the cognitive evolution of the child and his conceptualisation of time with the emergence of subordination between three and seven. For bilingual as well as monolingual children the first subordinations - and the earliest subordinators - are temporal and causal.

We then describe the bilingual linguistic development of these types of subordination, as organized into three phases:

1) paratactic structures without connector;

2) hypotactic structures with connectors - these phases are found with bilingual as well as monolingual children. For our subjects, this stage ends with the adequate expression of temporal and causal subordinates in French, whether it be the strong or the weak language of the child. In German however, word order and use of some connectors still diverge from adult grammar. Some children first produce a "sketch"or over-extension of the connector which links the two sentences. 


\section{AUTEUR}

\section{VERA FÜHRER-NICOD}

Université de Marne-la-Vallée Vera, andria@univ-mlv.fr 